\title{
Use of GFP-tagged Trichoderma harzianum as a tool to study the biological control of the potato cyst nematode Globodera pallida
}

\author{
J.B. Contina, L.M. Dandurand*, G.R. Knudsen
}

University of Idaho, Plant, Soil and Entomological Sciences Dept., 875 Perimeter Drive MS 2339, Moscow, ID, 83844-2339, United States

\section{A R T I C L E I N F O}

\section{Article history:}

Received 19 November 2016

Accepted 10 March 2017

Available online 31 March 2017

\section{Keywords:}

Biological control

Globodera pallida

Potato cyst nematode

Potato

Rhizosphere

Trichoderma harzianum

\begin{abstract}
A B S T R A C T
The fungus Trichoderma harzianum strain ThzID1-M3 isolated in the Palouse region of the state of Idaho and transformed to express green fluorescent protein (GFP) was used as a biomarker to study the biological control processes of the potato cyst nematode Globodera pallida. Experiments were conducted to evaluate the effect of T. harzianum ThzID1-M3 on G. pallida infection and reproduction at 45 and 75 days after inoculation respectively; and to observe, through microscopy rhizosphere chamber, the colonization of $G$. pallida second-stage juveniles and potato rhizoplane by T. harzianum ThzID1-M3 10 days after inoculation. Significant reduction of $G$. pallida infection in potato roots was observed when soil was amended with T. harzianum ThzID1-M3. Globodera pallida cysts recovered from soil and G. pallida reproduction rate were significantly reduced by $49 \%$ and $60 \%$ respectively, when soil was amended with $T$. harzianum ThzID1-M3 compared to the non-amended soil. Microscopic observations showed that $T$. harzianum ThzID1-M3 colonized G. pallida second-stage juveniles and cysts, and proliferated in the rhizoplane and rhizosphere of potato. To our knowledge, this is the first report on the study of Trichoderma spp. marked with GFP against a potato cyst nematode. The use of GFP-tagged T. harzianum has the potential to monitor the biological control processes of $G$. pallida and the tools advanced in this study should facilitate the design of novel strategies to control this economically important nematode pest of potato.
\end{abstract}

(c) 2017 Elsevier B.V. All rights reserved.

\section{Introduction}

The potato cyst nematode Globodera pallida Behrens (1975) and Stone (1972) is a globally regulated pest of potato (Solanum tuberosum). Globodera pallida is a quarantine pest in the state of Idaho, where it was first found in the United States in 2006 (Hafez et al., 2007). At high levels of infestation in a field, G. pallida can reduce tuber yields up to $80 \%$ (Talavera et al., 1998; Vasyutin and Yakovleva, 1998). Globodera pallida is characterized by the development of a globose cyst, which is the dead female body containing several hundred nematode eggs (Brodie et al., 1993). Cysts can survive in soil for many years (Perry, 1999) and the pest is usually spread over long distances by contaminated soils, tubers or by contaminated farm machinery (Evans and Stone, 1977).

Trichoderma harzianum, a soilborne filamentous fungus, has been extensively evaluated as a biocontrol agent and found effective against many soilborne plant pathogens (Bae and

\footnotetext{
* Corresponding author.

E-mail address: Imd@uidaho.edu (L.M. Dandurand).
}

Knudsen, 2000, 2006; Dandurand et al., 2000; Dandurand and Knudsen, 1993; Hadar et al., 1979; Harman et al., 2004; Knudsen et al., 1991; Knudsen and Bin, 1990; Sharon et al., 2001; Sivan et al., 1984; Weindling, 1932). Mechanisms of biocontrol used by $T$. harzianum against soilborne pathogens consist in: (1) mycoparasitism (Barak et al., 1985; Chet et al., 1981; Papavizas, 1985); (2) antibiosis and production of hydrolytic enzymes (Howell, 1998; Lorito et al., 1996); (3) competition (Papavizas, 1985); (4) root colonization (Altomare et al., 1999; Harman et al., 2004); or (5) plant growth promotion and induced resistance in host (Altomare et al., 1999; Howell et al., 2000; Kleifeld and Chet, 1992; Windham et al., 1986; Yedidia et al., 1999, 2000, 2003).

Trichoderma harzianum strain ThzID1 was obtained from Palouse silt loam soil on the University of Idaho Plant Science Farm in Moscow, Idaho (Knudsen and Bin, 1990) and has been reported to colonize sclerotia of Sclerotinia sclerotiorum (Knudsen et al., 1991; Bae and Knudsen, 2006; Kim and Knudsen, 2009). Globodera pallida reproduction rate was significantly reduced by $T$. harzianum ThzID1 during two consecutive greenhouse experiments (Dandurand and Knudsen, 2016). Bae and Knudsen (2000) transformed T. harzianum ThzID1 with three exogenous genes 
encoding green fluorescent protein (GFP), enabling rapid observation of its hyphae, mycelia and conidia. Both wild-type ( $T$. harzianum ThzID1) and GFP-transformed (T. harzianum ThzID1M3) strains colonized sclerotia of Sclerotinia sclerotiorum at levels above those of indigenous Trichoderma spp. in untreated controls, and there were no significant differences in colonization levels between wild-type and co-transformant strains; however, the presence of the GFP and GUS marker genes permitted differentiation of introduced Trichoderma from indigenous strains (Bae and Knudsen, 2000).

GFP is a powerful tool for monitoring bacterial and fungal activities in situ and does not require any substrate or additional cofactors to fluoresce (Bloemberg et al., 1997; Lorang et al., 2001; Lu et al., 2004). Orr and Knudsen (2004) demonstrated that the use of GFP-transformant of T. harzianum, along with epifluorescence microscopy, is a useful tool to distinguish active hyphal biomass, the form of the fungus that is functional for biological control, from inactive propagules such as conidia or chlamydospores that are enumerated by plate counts.

We hypothesized that the use of T. harzianum ThzID1-M3 would allow direct observation of the biocontrol processes of $G$. pallida. Trichoderma harzianum ThzID1-M3, as a biomarker, should reveal the mechanisms employed to reduce G. pallida infection and reproduction in potato. Biocontrol of G. pallida has been previously described using a wild-type strain (T. harzianum ThzID1) (Dandurand and Knudsen, 2016). This study was focused on elucidating the processes involved in the biocontrol of G. pallida using T. harzianum ThzID1-M3.

\section{Materials and methods}

\subsection{Propagation of G. pallida and T. harzianum ThzID1-M3}

Globodera pallida, obtained from an infested potato field in Shelley ID, was propagated on the susceptible potato cultivar 'Desiree' under greenhouse conditions of $18^{\circ} \mathrm{C} \pm 2{ }^{\circ} \mathrm{C}$ and 16:8-h light: dark period (Dandurand and Knudsen, 2016; Kooliyottil et al., 2016). After 16 weeks of growth, cysts were recovered from soil using the Fenwick method of flotation (Fenwick, 1940) and stored at $4{ }^{\circ} \mathrm{C}$ prior experimental use. The identity of $G$. pallida was confirmed by morphological and molecular methods (Skantar et al., 2007). Cysts, with 53\% hatching ability, 70\% viability and 304.2 eggs cyst ${ }^{-1}$, were surface-sterilized in a solution of $0.5 \%$ $\mathrm{NaOCl}$ for $5 \mathrm{~min}$ and rinsed thoroughly with sterile distilled water.

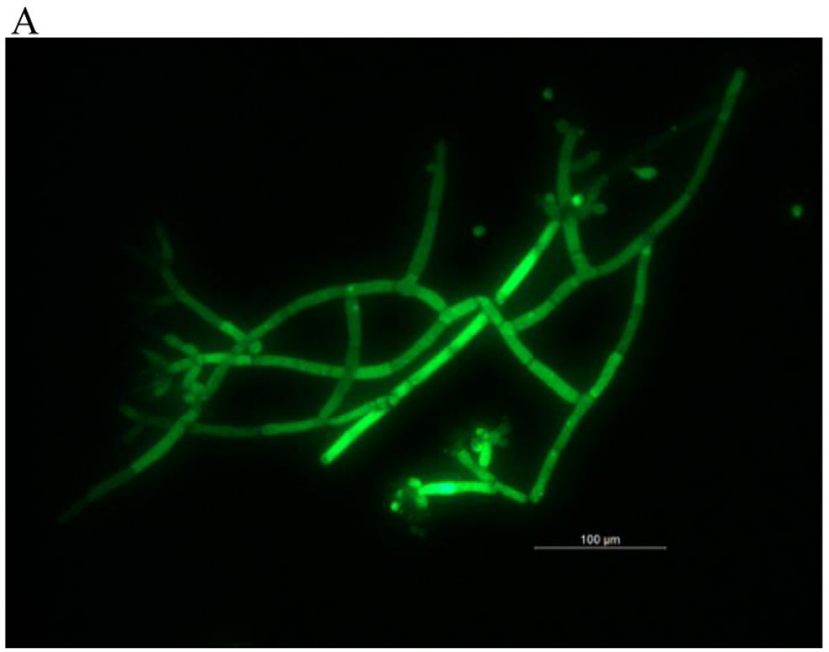

Ten cysts were put inside a sterile nylon mesh bags (McMaster Carr, Elmhurst, IL) with a $250 \mu \mathrm{m}$ of mesh opening. The nylon mesh was sealed along the edges with a hand sealer (Sealer 8" F-200, Sealer sales Inc., Northridge, CA), and were placed in sterilized distilled water for hydration for 3 days before amending to soil.

Trichoderma harzianum ThzID1-M3, isolated from Palouse silt loam soil on the University of Idaho Plant Science Farm in Moscow, ID (Knudsen and Bin, 1990) and transformed to express green fluorescent protein (GFP) (Bae and Knudsen, 2000), was maintained on potato dextrose agar (PDA) for 1 week at $22^{\circ} \mathrm{C}$. The identity of $T$. harzianum ThzID1-M3 was confirmed by morphological and molecular methods (Bae and Knudsen, 2000; Barnett and Hunter, 1998; Kim and Knudsen, 2008), and was observed under fluorescence microscopy (Leica DMI3000 B, Leica Microsystems, Wetzlar, Germany) for GFP activities (Fig. 1A). Oat kernels were used as growth substrate for T. harzianum ThzID1-M3. Twohundred grams of oat kernels placed in a 1000-ml Erlenmeyer flask were mixed with $200 \mathrm{ml}$ of distilled water and autoclaved twice at $121^{\circ} \mathrm{C}$. After $24 \mathrm{~h}$, the mixture was inoculated with five $T$. harzianum ThzID1-M3 fungal plugs (7-mm diameter) from PDA plate and was incubated at $22^{\circ} \mathrm{C}$ for 20 days prior to experimental use.

\subsection{Plant material culture and growth}

Potato tubers (S. tuberosum) cv. 'Russet Burbank', classified as certified disease free (from the Nuclear Potato Seed Program, University of Idaho), were used for experiments conducted in clay pots. Tubers were sterilized for $5 \mathrm{~min}$ in $0.5 \% \mathrm{NaOCl}$, rinsed thoroughly in sterile distilled water, dried, and left for 1 week under dark conditions to break dormancy prior to planting. For experiments conducted in microscopy rhizosphere chambers (Micro-Rocs), potato plants (S. tuberosum) cv. 'Russet Burbank' were grown from tissue culture plantlets in standard media (Murashige and Skoog, 1962) for 1 week prior to transplanting.

\subsection{Effect of T. harzianum ThzID1-M3 on G. pallida infection and reproduction in potato}

Experiments were conducted using Prosser fine sandy loam soil, which was air-dried and sieved through a 5-mm mesh. A 2:1 sand: soil mixture (56\% sand, $35 \%$ silt, $8 \%$ clay, $\mathrm{pH} 7.0$ ) was autoclaved twice for $90 \mathrm{~min}$ at $121^{\circ} \mathrm{C}$ prior to experimental use. A 15-cm diameter size Terra Cotta clay pot (The Home Depot, Atlanta,

B

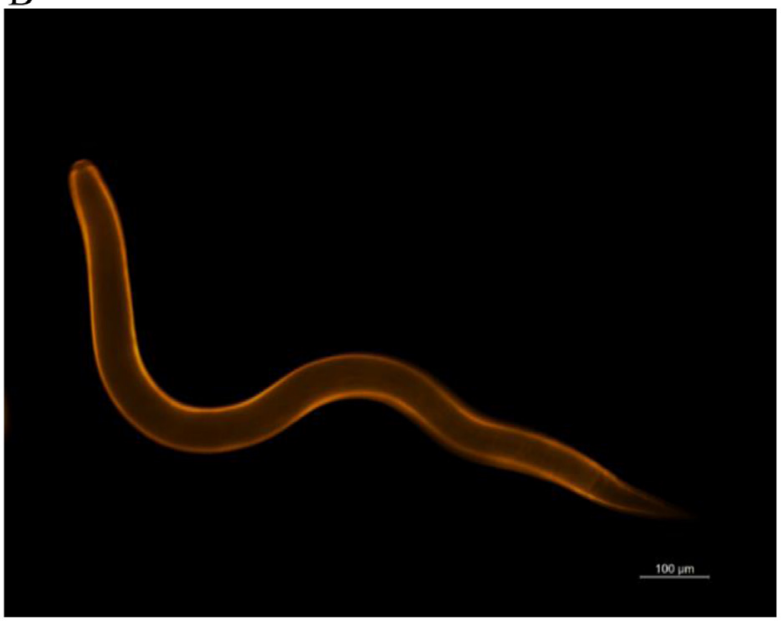

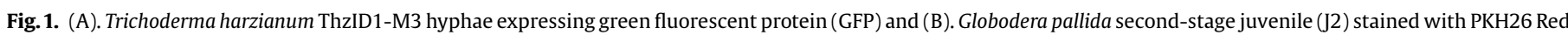

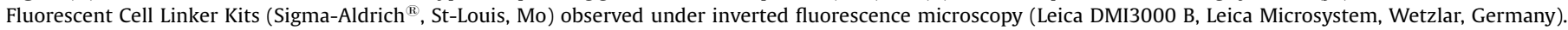


GA) was used for experiments, each clay pot contained $1.5 \mathrm{~kg}$ soil mix. Two experiments at 45 and 75 days were conducted to evaluate the effect of T. harzianum ThzID1-M3 on G. pallida infection and reproduction in potato roots. Proliferation of $T$. harzianum ThzID1-M3 in cysts, roots and soil, was also evaluated. There were two treatments for each time period: (1) the nonamended control consisted of G. pallida cysts applied to nonamended soil; and (2) G. pallida cysts applied to soil amended with T. harzianum ThzID1-M3. Treatments included five replicates in a completely randomized design. Five grams of oat kernels colonized by $T$. harzianum ThzID1-M3 fungal inoculum $\left(10^{6}\right.$ conidia $\mathrm{g}^{-1}$ oat kernel) was mixed into $1.5 \mathrm{~kg}$ of soil and distributed to a clay pot. A cyst bag was placed at 5-cm below the soil line and covered with 2$\mathrm{cm}$ soil before placing one potato tuber and covering with soil. Globodera pallida was applied at an initial rate (Pi) of $2.03 \mathrm{egg} \mathrm{g}^{-1}$ soil. Pots were maintained at $18{ }^{\circ} \mathrm{C} \pm 2{ }^{\circ} \mathrm{C}, 60 \%$ relative humidity, and 16:8-h light: dark period under greenhouse conditions. Pots were watered twice daily in the amount of $100 \mathrm{ml}$ of water, and fertilized three times a week using Jack's classic garden fertilizer 20-20-20 (JR Peters Inc., Allentown, PA) applied at a rate of $0.5 \mathrm{~g}$ liter ${ }^{-1}$ water.

\subsection{Microscopic observation of T. harzianum ThzID1-M3 biocontrol activity}

One-week-old tissue culture potato plantlets were transplanted into Micro-Rocs chambers (Advanced Science Tools, LLC, Pullman, WA), and were inserted between the cover glass and the filter paper, the adjacent slot was filled with soil consisted in $85 \%$ peat and organic materials mixture (Sunshine Mix, SunGro ${ }^{\circledR}$, Vancouver, Canada). Potato plantlets were left to grow for 2 weeks under greenhouse conditions at $18^{\circ} \mathrm{C} \pm 2{ }^{\circ} \mathrm{C}, 16$ :8 light: dark conditions, watered once daily with $25 \mathrm{ml}$ of water and fertilized three times a week at a rate of $0.5 \mathrm{gl}^{-1}$ of water using Jack's classic garden fertilizer 20-20-20 (JR Peters Inc., Allentown, PA). To obtain second stage hatched juveniles, 20 cysts were placed inside a hatching chamber, made of 30- $\mu$ m nylon mesh, attached to a 2-cm diameter plastic pipe and placed inside a corning centrifuge tube (SigmaAldrich $^{\mathbb{R}}$, St-Louis, Mo). Two milliliters of fresh sterilized water, $1 \mathrm{ml}$ of potato root diffusate (PRD), $1.5 \mathrm{mg} \mathrm{ml}^{-1}$ of gentamicin and $0.05 \mathrm{mg} \mathrm{ml}^{-1}$ of nystatin were added into the corning centrifuge tube, followed by incubation at $22^{\circ} \mathrm{C}$ for 2 weeks. Hatched juveniles (J2s) were cleaned of eggs shells and other debris by using sucrose gradient centrifugation (30-60\%) (Kooliyottil et al., 2016). Hatched J2 s were collected and incubated overnight in a solution containing $100 \mu \mathrm{g} \mathrm{ml}^{-1}$ of ampicillin and streptomycin $(\mathrm{w} / \mathrm{v})$ on a shaker at room temperature. Hatched J2s were further treated with benzethonium chloride $\left(0.125 \mathrm{~g} \mathrm{ml}^{-1}\right)$ (SigmaAldrich $^{\circledR}$, St-Louis, Mo) by continuous shaking at room temperature for 20 min on a rocker (Standard Analog Rocker, VWR, Radnor, PA); washed 8 times by centrifugation ( $4000 \mathrm{rpm}$ for $10 \mathrm{~min}$ ) and re-suspended in sterile distilled water (modified from Upadhyay et al., 2013). Collected J2s were stained using PKH26 Red Fluorescent Cell Linker Kits, $4 \times 10^{-6} \mathrm{M}$ (Sigma-Aldrich ${ }^{\circledR}$, St-Louis, Mo) according to manufacturer's protocol, and incubated for $10 \mathrm{~min}$ at room temperature in dark with intermediate shaking (3 times) for $15 \mathrm{~s}$ and washed 5 times in sterile distilled water. The stained J2s (Fig. 1B) were collected by centrifugation at $4000 \mathrm{rpm}$ for $10 \mathrm{~min}$ and transferred to $0.01 \%$ sterile agarose (modified from Sobczak et al., 2005), and kept at $-4{ }^{\circ} \mathrm{C}$ until use. Potato plantlets were inoculated with 1-week old T. harzianum ThzID1-M3 on PDA discs according to the treatment. Three 7-mm diameter PDA discs were crushed and mixed with sterile distilled water and pipetted over potato root surfaces through the Micro-Rocs cover glass at a rate $2 \mathrm{ml}$ containing $3.2 \times 10^{5}$ conidia $\mathrm{ml}^{-1}$. After 2 weeks, potato plantlets were inoculated with $100 \mu \mathrm{l}$ of agarose solution containing $2 \mathrm{~J} \mathrm{~s} \mu \mathrm{l}^{-1}$ (Kooliyottil et al., 2016). There were two treatments, as follows: (1) G. pallida J2s only applied to nonamended soil; and (2) G. pallida J2s applied to soil amended with the biocontrol T. harzianum ThzID1-M3. Treatments were arranged in a completely randomized design, replicated five times and the experiment was repeated. Inoculated plantlets were maintained under greenhouse conditions at $18^{\circ} \mathrm{C} \pm 2{ }^{\circ} \mathrm{C}, 16: 8$ light: dark conditions for 10 days, and were placed under inverted fluorescence microscopy (Leica DMI3000 B, Leica Microsystems, Wetzlar, Germany) at day 5 and 7 to observe the interactions of $T$. harzianum ThzID1-M3 with: (1) the potato rhizoplane; (2) G. pallida J2s; and (3) roots infected by G. pallida J2s.

\subsection{Nematode infection assessment}

Acid fuchsin (Sigma-Aldrich ${ }^{\circledR}$, St-Louis, Mo), an acidic dye, was used to stain G. pallida juveniles in potato roots in order to determine the level of root infections (Byrd et al., 1983) using a dissecting microscope (Leica M80, Leica Microsystems, Wetzlar, Germany). After counting, roots were dried and weighed in a scale (Thermo Fisher Scientific, Waltham, MA) and juvenile nematode density $\mathrm{g}^{-1}$ root was determined.

\subsection{Nematode reproduction assessment}

Cysts were extracted from soil using the Fenwick flotation method (Fenwick, 1940). Extracted cysts were crushed in $100 \mu \mathrm{l}$ of sterile distilled water and eggs were counted under a dissecting microscope (Leica M80, Leica Microsystems, Wetzlar, Germany). Nematode reproductive factor (Rf) was calculated based on the ratio of the final egg population density (Pf) over the initial egg population density $(\mathrm{Pi})$ per gram of soil.

\subsection{Trichoderma harzianum ThzID1-M3 proliferation assessment}

To assess the presence of T. harzianum ThzID1-M3 in cyst, potato rhizoplane and rhizosphere, molecular characterization of $T$. harzianum ThzID1-M3 was performed and consisted in DNA extractions followed by PCR analysis, culture media plating technique, and fluorescent microscopy observations of fungal colonization in G. pallida second-stage juveniles, cysts and potato rhizoplane.

DNA materials were extracted from root segments and cyst samples by using the DNeasy plant DNA extraction kit (Qiagen Inc., Venlo, Netherlands) according to manufacturer's protocol. DNA was extracted from soil samples using the PowerSoil DNA isolation kit (MoBio Laboratories Inc., Carlsbad, CA) according to manufacturer's protocol. DNA materials extracted from root, cyst, and soil samples was processed using EasyStart PCR Mix-in-a-Tube (Thermo Fisher Scientific Inc., Waltham, MA) according to manufacturer protocol. DNA samples were amplified through a C1000 Touch $^{\mathrm{TM}}$ Thermal Cycler (Bio-Rad Laboratories Inc., Hercules, CA). Primers for Trichoderma spp. were designed from GenBank (National Center for Biotechnology Information [NCBI], Bethesda, $\mathrm{MD}$ ) based on the conserved regions of the consensus sequence (Kim and Knudsen, 2008). Forward and Reverse primers were obtained from Sigma-Aldrich ${ }^{\circledR}$ (Sigma-Aldrich ${ }^{\circledR}$, St-Louis, Mo). Forward primer sequence (TGP4-F) was as follows: 5'CTCCCAAACCCAATGTGA AC-3'; Reverse primer sequence (TGP4R) was as follows: 5'-GCGAGTGTGCAAAAT ACTG-3'; and the amplicon size based on the consensus sequence was $466 \mathrm{bp}$.

To further assess the presence of T. harzianum ThzID1-M3, roots were sterilized in $0.5 \% \mathrm{NaOCl}$, washed thoroughly in sterile distilled water and cut into $1-\mathrm{cm}$ segment. Five $1-\mathrm{cm}$ root segments were plated in Trichoderma selective media (TSM) (Elad et al., 1981), replicated four times, and were incubated for 1 week 
at $22^{\circ} \mathrm{C}$. Globodera pallida cysts, from recovered cyst bags, were sterilized in $0.5 \% \mathrm{NaOCl}$ and washed thoroughly in sterile distilled water. Ten cysts were placed in a $1.5 \mathrm{ml}$ microtube and were crushed in $500 \mu \mathrm{l}$ sterile distilled water. Serial dilution method was used to quantify the presence of $T$. harzianum ThzID1-M3 at $1: 10^{3}, 1: 10^{4}, 1: 10^{5}$ and $1: 10^{6}$-fold dilution. Diluted solutions were spread in TSM and were incubated for 1 week at $22^{\circ} \mathrm{C}$. For rhizosphere assay, ten grams of soil samples were mixed in $90 \mathrm{ml}$ of sterile distilled water to yield a 10-fold dilution. One $\mathrm{ml}$ of the 10:1 dilution was added to $9 \mathrm{ml}$ of sterile distilled water to yield $1: 10^{3}$ to $1: 10^{6}$-fold dilution, were spread in TSM and incubated for 1 week at $22{ }^{\circ} \mathrm{C}$. Besides GFP activities, the production of conidia from effused conidiophores, or from conidiophores aggregated into fascicles or pustules was used as a morphological characteristic of Trichoderma spp. for identification in TSM-grown culture (Barnett and Hunter, 1998).

To visualize T. harzianum ThzID1-M3 proliferation in G. pallida second-stage juveniles, cysts and potato rhizoplane, an inverted fluorescence microscope (Leica DMI3000 B, Leica Microsystems, Wetzlar, Germany), equipped with a Leica DFC340 FX digital fluorescence camera, a Leica AF6000E fluorescence software, a rhodamine and a GFP filters, was used to observe T. harzianum ThzID1-M3 hyphae and mycelia.

\subsection{Statistical analysis}

All data analyses were performed using SAS package 9.4 (SAS Institute Inc., Cary, N.C.). Analysis of variance using generalized linear mixed effects procedure (Proc Glimmix) was performed to analyze root weight, G. pallida infection and reproduction in potato roots with significant differences occurring at level of $P \leq 0.05$. Means were separated by the Tukey-Kramer adjustment.

\section{Results}

\subsection{Nematode infection}

At 45 days post inoculation (DPI), average G. pallida infection in non-amended soil was 2.49 fourth-stage juveniles $(\mathrm{J} 4) \mathrm{g}^{-1}$ root, while in amended soil with T. harzianum ThzID1-M3 the infection level was $0.40 \mathrm{~J}^{-1}$ root, resulting in significant reduction in infection rate by $84 \%(P \leq 0.05)$ (Table 1$)$. Globodera pallida juveniles (second [J2], and third [J3] stage) and adult females were observed in the non-amended soil treatment, however they were not detected in soil amended with T. harzianum ThzID1-M3 (Table 1). Despite this decrease, no significant difference was observed in fresh potato root weight $(P \geq 0.05)$ (Table 1$)$. In the infection assay conducted in Micro-Rocs chambers, no interaction between treatment and experiment effects were detected, therefore experimental data from both experiments were combined. Average G. pallida J2s in non-amended control was 751.30 $\mathrm{J} \mathrm{sg}^{-1}$ root, while in amended treatment with $T$. harzianum ThzID1-M3 the average of G. pallida J2 s was $244.60 \mathrm{~J} 2 \mathrm{~s} \mathrm{~g}^{-1}$ root, resulting in significant reduction in infection rate by $67 \%(P \leq 0.05)$ (Table 2 ). Average potato dry root weight in non-amended soil was
Table 2

Influence of Trichoderma harzianum ThzID1-M3 on Globodera pallida second-stage juveniles (J2) root infection and root weight using Micro-Rocs chambers.

\begin{tabular}{lll}
\hline Treatment & Root weight $^{\mathrm{a}}(\mathrm{g})$ & G. pallida $\mathrm{J}^{\mathrm{g}^{-1}}$ root \\
\hline Non-amended control & 0.13 & 751.30 \\
T. harzianum ThzID1-M3 & 0.21 & 244.60 \\
\hline
\end{tabular}

Means of root weight and G. pallida $\mathrm{J} \mathrm{g}^{-1}$ root are significant $(P \leq 0.05)$ according to Tukey-Kramer adjustment test of the Glimmix procedure.

${ }^{a}$ Dry root weight.

$0.13 \mathrm{~g}$, while in amended soil with T. harzianum ThzID1-M3 dry root weight was $0.21 \mathrm{~g}$, resulting in significant increase in potato dry root weight by $62 \%(P \leq 0.05)$ (Table 2 ).

\subsection{Nematode reproduction}

At 75 days post inoculation (DPI), average final G. pallida egg density (Pf) was 6.40 eggs $^{-1}$ soil and reproduction rate (Pf/Pi) was 3.15 in non-amended soil. In soil amended with $T$. harzianum

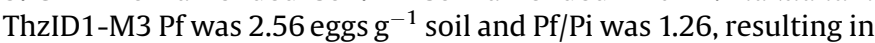
significant reduction in nematode reproduction by $60 \%(P \leq 0.05)$ (Table 3 ). Average number G. pallida cysts in non-amended soil was 55 cysts root $^{-1}$ ball, while in amended soil with $T$. harzianum ThzID1-M3 the average was 28 cysts root $^{-1}$ ball, resulting in significant reduction of $G$. pallida cysts by $49 \%(P \leq 0.05)$ (Table 3 ). No significant difference was observed in fresh potato root weight $(P \geq 0.05)$ (Table 3$)$ whether or not soil was amended with $T$. harzianum ThzID1-M3.

\subsection{Trichoderma harzianum ThzID1-M3 proliferation}

Electrophoresis analysis of PCR products revealed the presence of T. harzianum ThzID1-M3, visible by the occurrence of white band in agarose gel, in: (1) G. pallida cyst, where non-amended treatment was compared to amendment with $T$. harzianum ThzID1-M3 (Fig. 2A); (2) potato rhizoplane, where non-amended treatment was compared to amendment with $T$. harzianum ThzID1-M3 (Fig. 2B); and (3) potato rhizosphere, where amended treatment with $T$. harzianum ThzID1-M3 is shown (Fig. 2C). Through plate assay, G. pallida cysts were found to be heavily colonized by $T$. harzianum ThzID1-M3 at an average of $1.41 \times 10^{5}$ conidia forming unit per cyst. Fluorescent microscopic observations of $G$. pallida cyst surfaces revealed heavy presence of $T$. harzianum ThzID1-M3 hyphae and mycelia, indicating signs of fungal parasitism (Fig. 3A, B). However, despite the heavy colonization of cysts, no colonized eggs were observed in crushed cysts. Examinations of plate assay revealed that T. harzianum ThzID1-M3 colonized 10\% of the potato rhizoplane. Fluorescent microscopic observations using Micro-Rocs chambers revealed that $T$. harzianum ThzID1-M3 was able to extensively colonize the potato rhizoplane and establish hyphal growth inside the cortex of the root system (Fig. 3C). Trichoderma harzianum ThzID1-M3 was observed to colonize and kill G. pallida juveniles (J2) prior to root infection (Fig. 3D). Globodera pallida juveniles (J2) were observed inside infected potato root in non-amended treatment (Fig. 3E) but

Table 1

Influence of Trichoderma harzianum ThzID1-M3 on Globodera pallida juveniles root infection and root weight at 45 days.

\begin{tabular}{|c|c|c|c|c|c|}
\hline Treatment & Root weight $^{\mathrm{a}}(\mathrm{g})$ & G. pallida ${\mathrm{J} 2 \mathrm{~g}^{-1} \text { root }}$ & G. pallida $\mathrm{J} \mathrm{g}^{-1}$ root & G. pallida $\mathrm{J} \mathrm{g}^{-1}$ root & G. pallida adult female $\mathrm{g}^{-1}$ root \\
\hline Non-amended control & 57.80 & 0.02 & 0.20 & 2.49 & 0.05 \\
\hline T. harzianum ThzID1-M3 & 52.54 & 0 & 0 & 0.40 & 0 \\
\hline
\end{tabular}

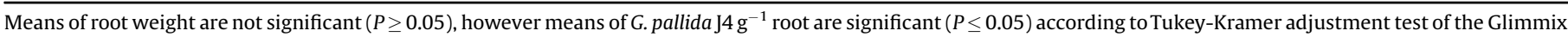
procedure.

a Fresh root weight. 
Table 3

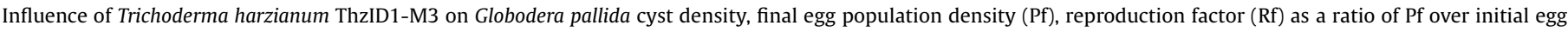
population density $(\mathrm{Pi})(\mathrm{Pi}=2.03)$, and root weight at 75 days.

\begin{tabular}{|c|c|c|c|c|c|}
\hline Treatment & Root weight $^{\mathrm{a}}(\mathrm{g})$ & Cyst root $^{-1}$ ball & Cystg $^{-1}$ soil & $\mathrm{Pfg}^{-1}$ soil & $\mathrm{Rf}$ \\
\hline Non-amended control & 59 & 55 & 0.04 & 6.40 & 3.15 \\
\hline T. harzianum ThzID1-M3 & 63 & 28 & 0.02 & 2.56 & 1.26 \\
\hline
\end{tabular}

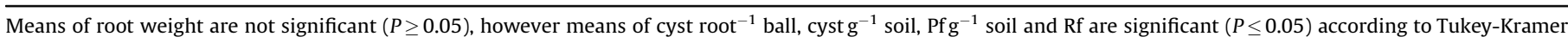
adjustment test of the Glimmix procedure.

a Fresh root weight.

not in roots colonized with $T$. harzianum ThzID1-M3. Through plate assay, potato rhizosphere was found to be heavily colonized by $T$. harzianum ThzID1-M3 at a rate of $10^{5} \mathrm{CFU} \mathrm{g}^{-1}$ soil.

\section{Discussion}

\subsection{Effect of T. harzianum ThzID1-M3 on G. pallida infection and reproduction}

Trichoderma harzianum ThzID1-M3 significantly reduced G. pallida infection and reproduction in potato roots. Similarly, amendment of soil with $T$. harzianum ThzID1 (non-transformed strain) reduced G. pallida reproduction rate by $43 \%$ and $48 \%$ during two consecutive potato crop cycles in greenhouse experiments (Dandurand and Knudsen, 2016). However, mechanisms behind such reduction in G. pallida reproduction rate were not fully understood. The use of T. harzianum ThzID1-M3 as a biomarker tool allows us to understand the interaction between the fungus and its target in order to define possible mechanisms behind the biocontrol of G. pallida in potato. Enabled with a constitutive GFP tag, which does not alter the biocontrol efficacy of the fungus, we have been able to track the physical presence of $T$. harzianum mycelia in G. pallida cysts, second-stage juveniles (J2), and in the rhizoplane and rhizosphere of potato. Trichoderma harzianum ThzID1-M3 was able to colonize both G. pallida cysts and secondstage juveniles (J2), however no egg colonization was observed. Colonization process was characterized by hyphal growth of $T$. harzianum toward G. pallida second-stage juveniles (J2), followed by surface contact and penetration of T. harzianum hyphae inside the nematode body resulting in larval death. Similarly, T. harzianum colonization process has been previously described in Globodera rostochiensis (Saifullah and Thomas, 1996; Trifonova, 2010), in
Meloidogyne javanica (Sahebani and Hadavi, 2008; Saifullah and Khan 2014; Sharon et al., 2001) and in M. incognita (Goswami et al., 2008). Other fungal species showed similar colonization process when applied against other plant-parasitic nematodes (Kiewnick and Sikora, 2006; Tobin et al., 2008; Zhang et al., 2014), and by expressing GFP in the fungus Clonostachys rosea, Zhang et al. (2008) showed the antagonist process of the saprophytic nematode Panagrellus redivivus started from the adherence of conidia to nematode cuticle for germination, followed by the penetration of germ tubes into the nematode body and subsequent degradation of the nematodes.

Although eggs were not observed to be colonized by $T$. harzianum ThzID1-M3, colonization of cysts could allow for close proximity of the biocontrol agent to the hatched juveniles, and lead to colonization of the infectious stage of the nematode prior to penetration into roots, as observed in the Micro-Rocs experiment. Potential mechanisms used by Trichoderma spp. to attack its prey that could be at play in the Micro-Rocs experiment include: (1) a positive chemotrophic growth towards its host; (2) direct contact with the prey; (3) formation of an appresorium-like penetration structures; and (4) production of tissue degrading hydrolytic enzymes that allows hyphal penetration and subsequent death of the prey, and its consumption by Trichoderma (Herrera-Estrella and Chet, 2004).

\subsection{Effect of T. harzianum ThzID1-M3 in the potato rhizoplane and rhizosphere}

Trichoderma harzianum ThzID1-M3 was able to colonize potato rhizoplane and rhizosphere. Under fluorescence microscopy, $T$. harzianum ThzID1-M3 was observed colonizing potato root surface and penetrating the root cortex. Rhizoplane colonization by $T$.
A

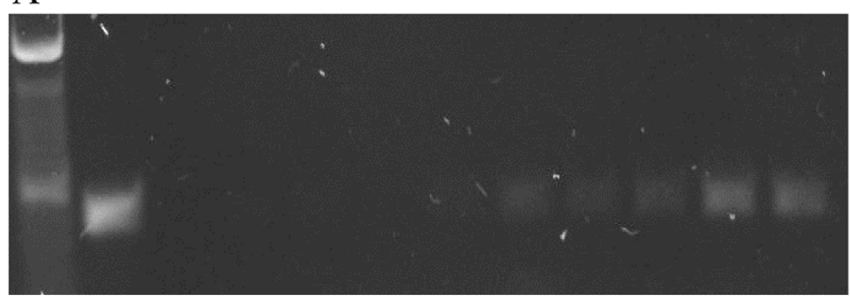

C
B

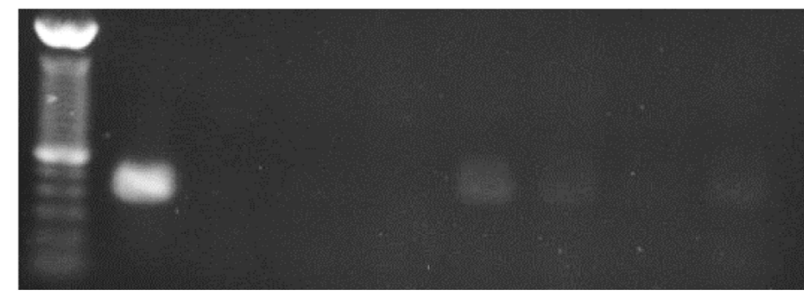

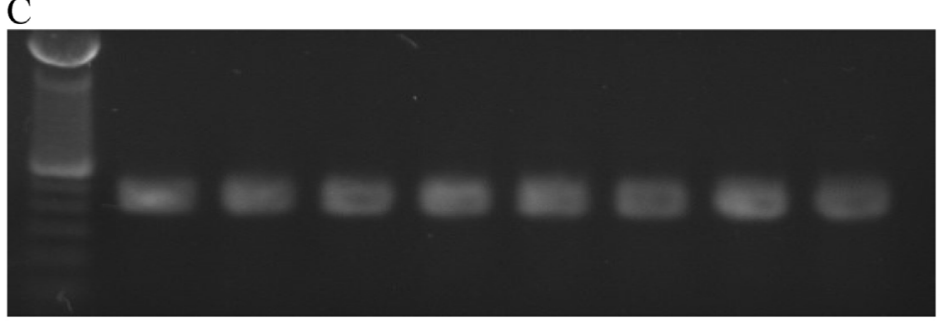

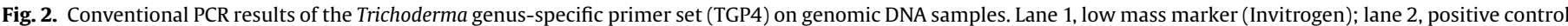

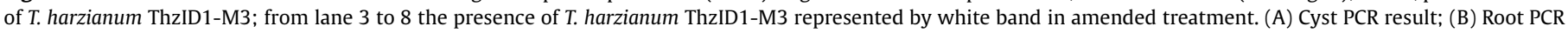
result; and (C) Soil PCR result. 

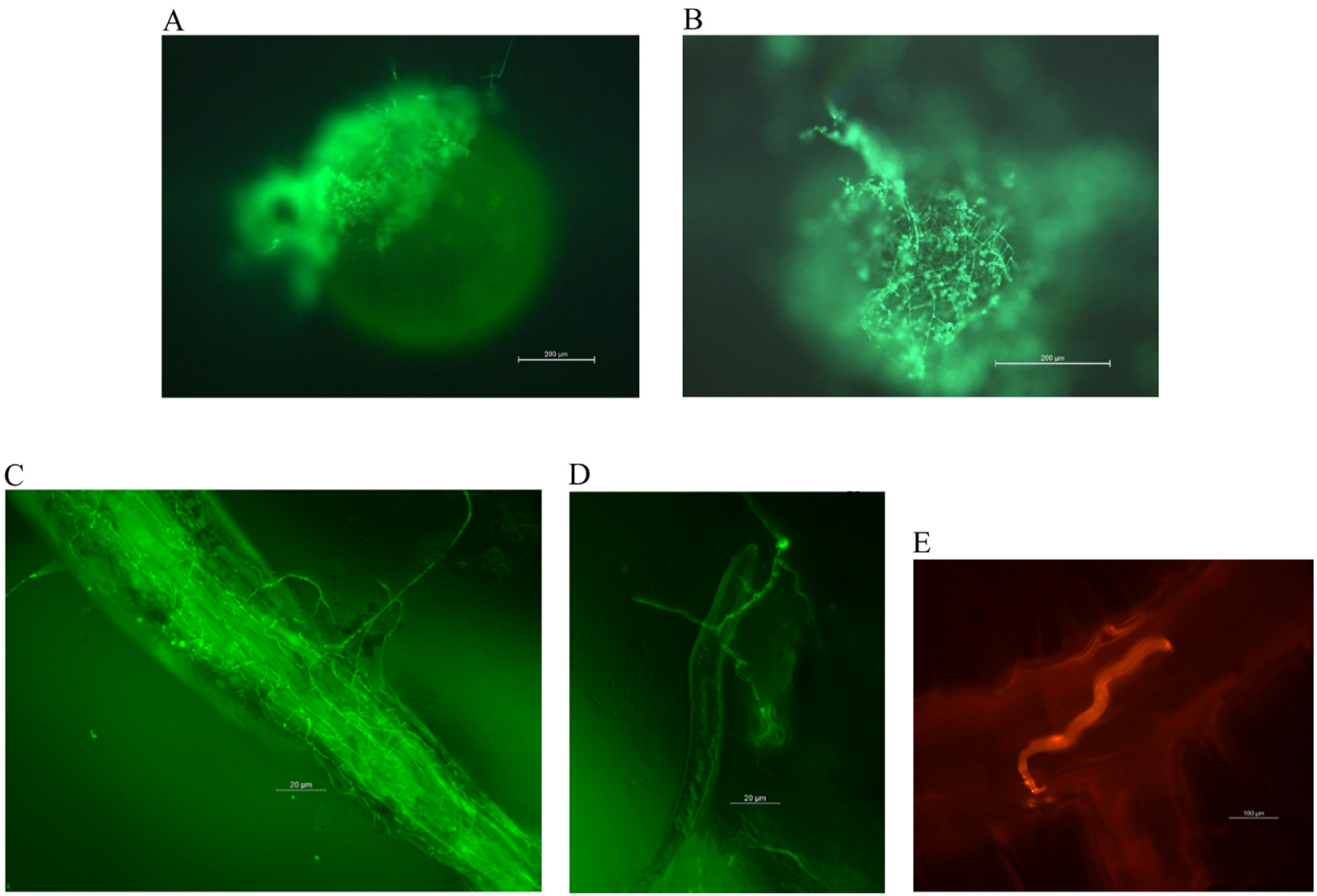

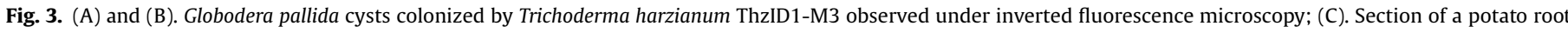

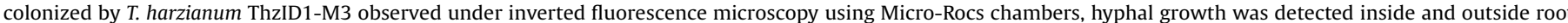

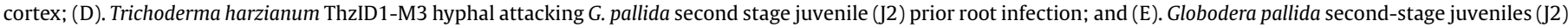
inside potato root observed under inverted fluorescence microscopy using Micro-Rocs chambers.

harzianum ThzID1-M3 contributes to the protection of the infection court and also favors T. harzianum ThzID1-M3 growth and proliferation in the rhizosphere, and the ability to colonize plant roots has often been stressed as an important requirement for Trichoderma spp. to act as biocontrol agents (Harman et al., 2004; Sivan and Chet, 1989). Previous studies described similar rhizoplane and rhizosphere colonization by T. harzianum (Ahmad and Baker, 1987; Chao et al., 1986; Orr and Knudsen, 2004). Lu et al. (2004) showed the GFP-tagged Trichoderma was able to colonize the outer layer of the roots of cucumber seedlings, as well as inoculated soil. Harman (2006) showed that Trichoderma spp. can colonize the root epidermis and outer cortical layers and increased plant growth and nutrient uptake, as we observed in colonization assays using Micro-Rocs chambers. Other studies suggest potential induction of plant systemic resistance triggered by the presence of Trichoderma spp. in plant roots leading to a broad spectrum protection against plant pathogens (Yedidia et al., 1999, 2000, 2003).

\section{Conclusions}

Trichoderma harzianum ThzID1-M3 was found to be useful to monitor the biocontrol interactions between $T$. harzianum and $G$. pallida. These are the first findings on the biocontrol process of $T$. harzianum marked with GFP on G. pallida. Trichoderma harzianum was able to reduce $G$. pallida infection and reproduction in potato roots through hyphal colonization of cysts and juveniles, although no colonization of eggs was observed. Trichoderma harzianum was also able to establish hyphal colonization in the potato rhizoplane and rhizosphere, possibly providing long term protection of the infection court. The tools advanced in this study should help develop an effective method to investigate the ecological properties of $T$. harzianum against plant pathogenic microorganisms.

\section{Acknowledgements}

The authors acknowledge USDA-NIFA Methyl Bromide Transition Program award number 2013-51102-21015 and the Idaho Potato Commission award number 12618 for providing funding for this research.

\section{References}

Ahmad, J.S., Baker, R., 1987. Rhizosphere competence of Trichoderma harzianum. Phytopathology 77, 182-189.

Altomare, C., Norvell, W.A., Bjorkman, T., Harman, G.E., 1999. Solubilization of phosphates and micronutrients by the plant-growth-promoting and biocontrol fungus Trichoderma harzianum Rifai 1295-22. Appl. Environ. Microbiol. 65, 2926-2933.

Bae, Y.S., Knudsen, G.R., 2000. Cotransformation of Trichoderma harzianum with 3 glucuronidase and green fluorescent protein genes provides a useful tool for monitoring fungal growth and activity in natural soil. Appl. Environ. Microbiol. 66, 810-815.

Bae, Y.S., Knudsen, G.R., 2006. Effect of sclerotial distribution pattern of Sclerotinic sclerotiorum on biocontrol efficacy of Trichoderma harzianum. Appl. Soil Ecol. 35 21-24.

Barak, R., Elad, Y., Mirelman, D., Chet, I., 1985. Lectins: a possible basis for specific recognition in the interaction of Trichoderma and Sclerotium rolfsii. Phytopathology 75, 458-462.

Barnett, H.L., Hunter, B.B., 1998. 4th ed. Illustrated Genera of Imperfect Fungi, vol. 92 American Phytopathological Society, St. Paul, MN.

Behrens, E., 1975. Globodera Skarbilovic, 1959, eine selbstaendige Gattung in der Unterfamilie Heteroderinae Skarbilovic, 1947 (Nematoda: Heteroderidae). 
Vortragstagung zu aktuellen Problemen der Phytonematologie am 29/5/1975 in Rostock. Manuskriptdruck der Vortraege. Rostock 1975, 12-26.

Bloemberg, G.V., O’Toole, G.A., Lugtenberg, B.J.J., Kolter, R., 1997. Green fluorescent protein as a marker for Pseudomonas spp. Appl. Environ. Microbiol. 63, 45434551.

Brodie, B.B., Evans, K., Franco, F., 1993. Nematode parasites of potatoes. In: Evans, K., Trudgill, D.L., Webster, J.M. (Eds.), Plant-Parasitic Nematodes in Temperate Agriculture. CAB International, Wallingford, pp. 87-132.

Byrd, D.W., Kirkpatrick, T., Barker, K.R., 1983. An improved technique for clearing and staining plant tissue for detection of nematodes. J. Nematol. 14, 142-143.

Chao, W.L., Nelson, E.B., Harman, G.E., Hoch, H.C., 1986. Colonization of the rhizosphere by biological control agents applied to seeds. Phytopathology 76, 60-65.

Chet, I., Harman, G.E., Baker, R., 1981. Trichoderma hamatum: its hyphal interactions with Rhizoctonia solani and Pythium spp. Microb. Ecol. 7, 29-38.

Dandurand, L.M., Knudsen, G.R., 1993. Influence of Pseudomonas fluorescens on hyphal growth and biocontrol activity of Trichoderma harzianum in the spermosphere and rhizosphere of pea. Phytopathology 83, 265-270.

Dandurand, L.M., Knudsen, G.R., 2016. Effect of the trap crop Solanum sisymbrifolium and two biocontrol fungi on reproduction of the potato cyst nematode, Globodera pallida. Ann. Appl. Biol. 169, 180-189.

Dandurand, L.M., Mosher, R.D., Knudsen, G.R., 2000. Combined effects of Brassica napus seed meal and Trichoderma harzianum on two soilborne plant pathogens. Can. J. Microbiol. 46, 1051-1057.

Elad, Y., Chet, I., Henis, Y., 1981. A selective medium for improving quantitative isolation of Trichoderma spp. from soil. Phytoparasitica 9, 59-67.

Evans, K., Stone, A.R., 1977. A review of the distribution and biology of the potato cyst nematodes Globodera rostochiensis and G. pallida. Int. J. Pest Manag. 23, 178-189.

Fenwick, D.W., 1940. Methods for the recovery and counting of cysts of Heterodera schachtii from soil. J. Helminthol. 18, 155-172.

Goswami, J., Pandey, R.K., Tewari, J.P., Goswami, B.K., 2008. Management of rootknot nematode on tomato through application of fungal antagonists, Acremonium strictum and Trichoderma harzianum. J. Environ. Sci. Health B 43, 237-240.

Hadar, Y., Chet, I., Henis, Y., 1979. Biological control of Rhizoctonia solani damping-off with wheat bran culture of Trichoderma harzianum. Phytopathology 69, 64-68.

Hafez, S.L., Sundararaj, P., Handoo, Z.A., Skantar, L., Carta, K., Chitwood, D.J., 2007. First report of the pale cyst nematode Globodera pallida, in the United States. Plant Dis. 91, 325.

Harman, G.E., Howell, C.R., Viterbo, A., Chet, I., Lorito, M., 2004. Trichoderma species: opportunistic, avirulent plant symbionts. Nat. Rev. 2, 43-56.

Harman, G.E., 2006. Overview of mechanisms and uses of Trichoderma spp. Phytopathology 190-194.

Herrera-Estrella, A., Chet, I., 2004. The biological control agent Trichoderma: from fundamentals to applications. In: Arora, D.K. (Ed.), Handbook of Fungal Biotechnology. Kluwer Academic Publishers, New York, pp. 1000-1020.

Howell, C.R., Hanson, L.E., Stipanovic, R.D., Puckhaber, L.S., 2000. Induction of terpenoid synthesis in cotton roots and control of Rhizoctonia solani by seed treatment with Trichoderma virens. Phytopathology 90, 248-252.

Howell, C.R., 1998. The role of antibiosis in biocontrol. In: Harman, G.E., Kubicek, C.P. (Eds.), Trichoderma and Gliocladium. Taylor \& Francis, London, pp. 173-184.

Kiewnick, S., Sikora, R.A., 2006. Biological control of the root-knot nematode Meloidogyne incognita by Paecilomyces lilacinus strain 251. Biol. Control 38, 179187.

Kim, T.G., Knudsen, G.R., 2008. Quantitative Real-Time PCR effectively detects and quantifies colonization of sclerotia of Sclerotinia sclerotiorum by Trichoderma spp. Appl. Soil Ecol. 40, 100-108.

Kim, T.G., Knudsen, G.R., 2009. Colonization of Sclerotinia sclerotiorum sclerotia by a biocontrol isolate of Trichoderma harzianum, and effects on myceliogenic germination. Biocontrol Sci. Technol. 19, 1081-1085.

Kleifeld, O., Chet, I., 1992. Trichoderma harzianum: Interaction with plants and effects on growth response. Plant Soil 144, 267-272.

Knudsen, G.R., Bin, L., 1990. Effects of temperature, soil moisture, and wheat bran on growth of Trichoderma harzianum from alginate pellets. Phytopathology 80 , 724-727.

Knudsen, G.R., Eschen, D.J., Dandurand, L.M., Bin, L., 1991. Potential for biocontrol of Sclerotinia sclerotiorum through colonization of sclerotia by Trichoderma harzianum. Plant Dis. 75, 446-470.

Kooliyottil, R., Dandurand, L.M., Govindan, B.N., Knudsen, G.R., 2016. Microscopy method to compare cyst nematode infection of different plant species. Adv. Biosci. Biotechnol. 7, 311-318.

Lorang, J.M., Tuori, R.P., Martinez, J.P., Sawyer, T.L., Redman, R.S., Rollins, J.A., Wolpert, T.J., Johnson, K.B., Rodriguez, R.J., Dickman, M.B., Ciuffetti, L.M., 2001 Green fluorescent protein is lighting up fungal biology. Appl. Environ. Microbiol. 67, 1987-1994.
Lorito, M., Farkas, V., Rebuffat, S., Bodo, B., Kubicek, C.P., 1996. Cell wall synthesis is a major target of mycoparasitic antagonism by Trichoderma harzianum. J. Bacteriol. 178, 6382-6385.

Lu, Z., Tombolini, R., Woo, S., Zeilinger, S., Lorito, M., Jansson, J.K., 2004. In vivo study of Trichoderma-pathogen-plant interactions using constitutive and inducible green fluorescent protein reporter systems. Appl. Environ. Microbiol. 70, 30733081.

Murashige, T., Skoog, F., 1962. A revised medium for rapid growth and bio assays with tobacco tissue cultures. Physiol. Plant. 15, 473-497.

Orr, K.A., Knudsen, G.R., 2004. Use of green fluorescent protein and image analysis to quantify proliferation of Trichoderma harzianum in nonsterile soil. Phytopathology 94, 1383-1389.

Papavizas, G.C., 1985. Trichoderma and Gliocladium: biology, ecology, and potential for biocontrol. Annu. Rev. Phytopathology 23, 23-54.

Perry, R.N., 1999. Desiccation survival of parasitic nematodes. Parasitology 119, 1930.

Sahebani, N., Hadavi, N., 2008. Biological control of the root-knot nematode Meloidogyne javanica by Trichoderma harzianum. Soil Biol. Biochem. 40, 20162020.

Saifullah, A., Khan, N.U., 2014. Low temperature scanning electron microscopic studies on the interaction of Globodera rostochiensis Woll. and Trichoderma harzianum Rifai. Pak. J. Bot. 46, 357-361.

Saifullah, A., Thomas, B.J., 1996. Studies on the parasitism of Globodera rostochiensis by Trichoderma harzianum using low temperature scanning electron microscopy. Afro-Asian J. Nematol. 6, 117-122.

Sharon, E., Bar-Eyal, M., Chet, I., Herrera-Estrella, A., Kleifeld, O., Spiegel, Y., 2001. Biological control of the root-knot nematode Meloidogyne javanica by Trichoderma harzianum. Phytopathology 91, 687-693.

Sivan, A., Chet, I., 1989. The possible role of competition between Trichoderma harzianum and Fusarium oxysporum on rhizosphere colonization. Phytopathology 79, 198-203.

Sivan, A., Elad, Y., Chet, I., 1984. Biological control effects of a new isolate of Trichoderma harzianum on Pythium aphanidermatum. Phytopathology 74, 498501.

Skantar, A.M., Handoo, Z.A., Carta, L.K., Chitwood, D.J., 2007. Morphological and molecular identification of Globodera pallida associated with potato in Idaho. J. Nematol. 39, 133-144.

Sobczak, M., Avrova, A., Jupowicz, J., Phillips, M.S., Ernst, K., Kumar, A., 2005. Characterization of susceptibility and resistance responses to potato cyst nematode (Globodera spp.) infection of tomato lines in the absence and presence of the broad-spectrum nematode resistance Hero gene. Mol. Plant Microbe Interact. 18, 158-168.

Stone, A.R., 1972. The round cyst species of Heterodera as a group. Ann. Appl. Biol. 71, $280-283$.

Talavera, M., Andreu, M., Valor, H., Tobar, A., 1998. Nematodos fitoparásitos en áreas productoras de patata de Motril y Salobreña. Investig. Agric. Prod. Prot. Veg. 13, 87-95.

Tobin, J.D., Haydock, P.P.J., Hare, M.C., Woods, S.R., Crump, D.H., 2008. Effect of the fungus Pochonia chlamydosporia and fosthiazate on the multiplication rate of potato cyst nematodes (Globodera pallida and G. rostochiensis) in potato crops grown under UK field conditions. Biol. Control 46, 194-201.

Trifonova, Z.T., 2010. Studies on the efficacy of some bacteria and fungi for control of Globodera rostochiensis. J. Agric. Sci. 55, 37-44.

Upadhyay, D., Kooliyottil, R., Mandjiny, S., Inman III, F.L., Holmes, L.D., 2013. Mass production of the beneficial nematode Steinernema carpocapsae utilizing a fedbatch culturing process. eSci. J. Plant Pathol. 2, 52-58.

Vasyutin, A.S., Yakovleva, V.A., 1998. Globodera in potatoes in Russia. Kartofel' i Ovoshchi 6, 29-32.

Weindling, R., 1932. Trichoderma lignorum as a parasite of other soil fungi. Phytopathology 22, 837-845.

Windham, M.T., Elad, Y., Baker, R., 1986. A mechanism for increased plant growth induced by Trichoderma spp. Phytopathology 76, 518-521.

Yedidia, I., Benhamou, N., Chet, I., 1999. Induction of defense responses in cucumber plants (Cucumis sativus L.) by the biocontrol agent Trichoderma harzianum. Appl. Environ. Microbiol. 65, 1061-1070.

Yedidia, I., Benhamou, N., Kapulnik, Y., Chet, I., 2000. Induction and accumulation of PR proteins activity during early stages of root colonization by the mycoparasite Trichoderma harzianum strain T-203. Plant Physiol. Biochem. 38, 863-873.

Yedidia, I., Shoresh, M., Kerem, Z., Benhamou, N., Kapulnik, Y., Chet, I., 2003. Concomitant induction of systemic resistance to Pseudomonas syringae pv. lachrymans in cucumber by Trichoderma asperellum (T-203) and the accumulation of phytoalexins. Appl. Environ. Microbiol. 69, 7343-7353.

Zhang, L., Yang, J., Niu, Q., Zhao, X., Ye, F., Liang, L., Zhang, K.Q., 2008. Investigation on the infection mechanism of the fungus Clonostachys rosea against nematodes using the green fluorescent protein. Appl. Microbiol. Biotechnol. 78, 983-990.

Zhang, S., Gan, Y., Xu, B., Xue, Y., 2014. The parasitic and lethal effects of Trichoderma longibrachiatum against Heterodera avenae. Biol. Control 72, 1-8. 